MODELING, IDENTIFICATION AND CONTROL, 1992, VOL. 13, NO. 3, 127-140

doi:10.4173/mic.1992.3.1

\title{
Multiple model estimation with inter-residual distance feedback
}

\author{
EIVIND J. LUND $\dagger$, JENS G. BALCHEN and BJARNE A. FOSS
}

Keywords: Adaptive systems, estimation, Kalman filters, parameter estimation, state estimation, system identification.

This paper presents a modification of the Multiple Model Adaptive Estimation concept. A trade-off problem between tracking the process and distinguishing the models is pointed out and an adaption of the elemental filters is proposed. The adaption scheme modifies the filters such that the predicted measurements do not become too close in some sense. This has considerable influence on the distinguishability of the filters and thereby the properties of the Multiple Model Adaptive Estimation algorithm. Stability of the method is considered, and a simulated example demonstrates the method.

\section{Introduction}

Multiple Model Adaptive Estimation (Anderson and Moore 1979, Athans and Chang 1976, Maybeck 1982), have been developed for simultaneous estimation of states and parameters in dynamic systems by Magill (1965) and further refined by Lainiotis (1971). A common assumption in MMAE is that the parameters only take on a finite number of different values. This is often an approximation of the continuous parameter case, but when prior knowledge indicates that the parameters only obtain a finite number of different values, MMAE is a method to utilize this information.

The MMAE concept constitutes a bank of state estimators running in parallel, producing one residual for each filter, as illustrated in Fig. 1. Each filter has a model that is different from the others and used to compute weighting coefficients which indicates the validity of each filter. These weighting coefficients are used to compute an

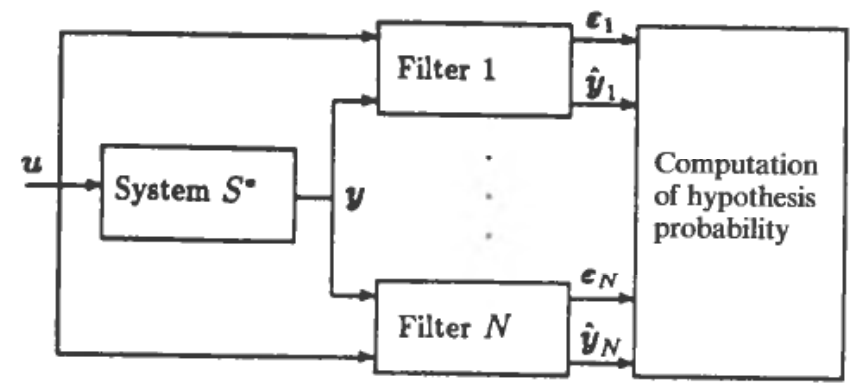

Figure 1. Multiple Model Adaptive Estimator.

Received 20 July 1991.

$\dagger$ The Norwegian Institute of Technology, Division of Engineering Cybernetics, N-7034 Trondheim, Norway.

This paper was presented at the 9th IFAC/IFORS Symposium on Identification and System Parameter Estimation, 8-12 July 1991, Budapest, Hungary. Reprinted with permission from IFAC. 
overall state estimate and parameter estimate. The MMAE concept has also been closely tied to adaptive control (Multiple Model Adaptive Control) by use of LQG controllers together with the overall state estimate (Athans et al. 1977, Magill 1965). Successful operation of MMAE is highly dependent upon the distinguishability of the models and tuning of the filters (Maybeck 1982, Maybeck and Pogoda 1989). When Kalman filters are used in the bank, there is feedback from the predicted measurements which may force the residuals too close together and interrupt the discrimination property of the filter bank.

In this paper a method, Inter-Residual Distance Feedback (IRDF), for on-line modification of the filters is proposed. The objective is to maintain the discrimination property of the filter bank. This is achieved by detuning the filters through modulation of certain filter parameters. The modulation is governed by a scalar quantity computed from a distance measure between the residuals.

The remaining part of this paper is organized as follows. In Section 2 the Multiple Model Adaptive Estimation method is briefly discussed. Section 3 highlights a trade-off problem in the MMAE concept. Namely that of discrimination versus tracking. Recognition of this problem leads on to the method of Inter-Residual Distance Feedback proposed in Section 4 which also addresses the stability of IRDF. The properties of our method are demonstrated by simulations in Section 5. Section 6 concludes the paper.

\section{The MMAE method}

Let $\boldsymbol{\theta}$ denote a $q$-dimensional vector of uncertain parameters in a dynamic stochastic state space model for a dynamic system. Assume that $\theta$ can take on only one of $N$ different values, $\theta_{i}, i=1, \ldots, N$. In this paper an operational mode $S_{i}$ is associated with $\theta_{i}$. Then the true system denoted $S^{*}$ is contained in the set $\mathscr{S}=\left\{S_{1}, \ldots, S_{N}\right\}$. The $i$ th operational mode $S_{i}$ is modeled as

$$
\begin{aligned}
\frac{d}{d t} x_{i}(t) & =f\left[x_{i}(t), u(t), \theta_{i}\right]+v_{i}(t) \\
y(t) & =g\left[x_{i}(t), \theta_{i}\right]+w(t)
\end{aligned}
$$

where $y(t)$ is measurement vector, $x_{i}(t)$ is state vector and $u(t)$ is measurable control input. The process noise $v_{i}(t)$ and measurement noise $w(t)$ are both assumed to be independent zero mean Gaussian with covariance matrices $V_{i}$ and $W$ respectively. Vector dimensions are: $\operatorname{dim} x_{i}(t)=\operatorname{dim} v_{i}(t)=n, \operatorname{dim} u(t)=r, \operatorname{dim} w(t)=\operatorname{dim} y(t)=m$. The functions $f[\cdot]$ and $g[\cdot]$ may be general non-linear and vector-valued. The model in Eqn. (1) is denoted by $M_{i}$ and within the limitations of modeling, $M_{i}$ describes the system $S^{*}$ when operating in mode $i$. All the models constitute a set $\mathscr{M}$ $=\left\{M_{1}, M_{2}, \ldots, M_{N}\right\}$.

At discrete time instants $t_{k}$, the MMAE algorithm calculates the probability $P_{i}\left(t_{k}\right)$ of each model $M_{i}$ conditioned on the discrete time measurement history $Y_{k}$ $=\left\{y\left(t_{1}\right), \ldots, y\left(t_{k}\right)\right\}$ obtained by sampling of $y(t)$

$$
P_{i}\left(t_{k}\right) \stackrel{\text { def }}{=} \operatorname{prob}\left\{S^{*}=M_{i} \mid Y_{k}\right\}
$$


$P_{i}\left(t_{k}\right)$ updates recursively as

$$
P_{i}\left(t_{k}\right)=\frac{p\left[y\left(t_{k}\right) \mid M_{i}, Y_{k-1}\right] P_{i}\left(t_{k-1}\right)}{\sum_{j=1}^{N} p\left[y\left(t_{k}\right) \mid M_{j}, Y_{k-1}\right] P_{j}\left(t_{k-1}\right)}
$$

where $p\left[y\left(t_{k}\right) \mid M_{i}, Y_{k-1}\right]$ is the density of $y\left(t_{k}\right)$ conditioned on $M_{i}$ and $Y_{k}$. The overall state estimate for the filter bank is given as

$$
\hat{\boldsymbol{x}}\left(t_{k}^{+}\right) \stackrel{\operatorname{def}}{=} \sum_{i=1}^{N} \hat{x}_{i}\left(t_{k}^{+}\right) P_{i}\left(t_{k}\right)
$$

where $\hat{x}_{i}\left(t_{k}^{+}\right)$is the state vector estimate in the $i$ th elemental filter after the measurement update, (Maybeck 1979). An estimate $\hat{\theta}\left(t_{k}\right)$, of $\theta$ is the conditional mean

$$
\hat{\theta}\left(t_{k}\right) \stackrel{\text { def }}{=} \sum_{i=1}^{N} \theta_{i} P_{i}\left(t_{k}\right)
$$

The Eqns. (3), (4) and (5) are valid for general models $M_{i}$ and conditional densities $p\left[y\left(t_{k}\right) \mid M_{i}, Y\left(t_{k-1}\right)\right]$. However due to filter complexity, applications of MMAE have mainly dealt with linear models and Kalman filters. With the assumption of Gaussian noise and linear models, the conditional densities are

$$
\begin{aligned}
p\left[y\left(t_{k}\right) \mid M_{i}, Y\left(t_{k-1}\right)\right] & =(2 \pi)^{-m / 2}\left(\operatorname{det}\left[\mathscr{E}_{i}\left(t_{k}\right)\right]\right)^{-1 / 2} \exp \left(-v_{i}\left(t_{k}\right)\right) \\
v_{i}\left(t_{k}\right) & =\frac{1}{2} \varepsilon_{i}^{\mathrm{T}}\left(t_{k}\right) \mathscr{E}_{i}^{-1}\left(t_{k}\right) \varepsilon_{i}\left(t_{k}\right)
\end{aligned}
$$

where $\varepsilon_{i}\left(t_{k}\right)=y\left(t_{k}\right)-\hat{y}_{i}\left(t_{k}\right)$ is the residual vector of the $i$ th elemental filter and $\mathscr{E}_{i}\left(t_{k}\right)$ is the estimated covariance matrix of $\varepsilon_{i}\left(t_{k}\right)$ at time instant $t_{k}$. For brevity the Kalman filter including its model $M_{i}$ is denoted $\mathscr{F}\left(M_{i}, K_{i}\right)$. Both $\varepsilon_{i}\left(t_{k}\right)$ and $\mathscr{E}_{i}\left(t_{k}\right)$ are provided by $\mathscr{F}\left(M_{i}, K_{i}\right)$. The Kalman filter equations for discrete time measurement update may be found in (Gelb 1984, Jazwinski 1970, Maybeck 1979, Maybeck 1982) for linear and nonlinear models.

When $S^{*}=M_{j}$ one should expect that

$$
v_{j}\left(t_{k}\right) \ll v_{i}\left(t_{k}\right), \forall i \neq j
$$

which is denoted as regular behaviour of the residuals. Now $P_{j}\left(t_{k}\right)$ increases towards unity while probabilities of the mismatched filters will decrease towards zero if the condition of Eqn. (7) persists over several measurements. A more formal convergence condition is given in (Anderson and Moore 1979). If $S^{*} \notin \mathscr{M}$ and/or the filters are tuned improperly it is possible that

$$
v_{1}\left(t_{k}\right) \approx v_{2}\left(t_{k}\right) \approx \ldots \approx v_{N}\left(t_{k}\right)
$$

The $P_{i}$ is now governed by $\operatorname{det}\left[\mathscr{E}_{i}\left(t_{k}\right)\right], i=1, \ldots, N$ and $P_{j}\left(t_{k}\right)$ increases if $\operatorname{det}\left[\mathscr{E}_{j}\left(t_{k}\right)\right]<\operatorname{det}\left[\mathscr{E}_{i}\left(t_{k}\right)\right], i \neq j$, while $P_{i}\left(t_{k}\right), i \neq j$ decrease. For Kalman filters and Extended Kalman filters $\operatorname{det}\left[\mathscr{E}_{i}\left(t_{k}\right)\right]$ is not dependent on which model is correct and erroneous decisions upon the valid model may result (Athans and Chang 1976). Hence the situation of Eqn. (8) is undesirable. The behaviour of MMAE as outlined above is related to the identifiability concept of the algorithm and tuning of the filters.

When using MMAE with changing parameters, a widely used ad hoc modification is to fix a lower bound on $P_{i}\left(t_{k}\right)$. Without such a lower bound it is seen from Eqn. (3) that a change will not be detected. A reasonable lower bound on $P_{i}$ is 0.001 which gives $P_{i}\left(t_{k}\right) \in[0 \cdot 001,1-(N-1) 0 \cdot 001]$. 


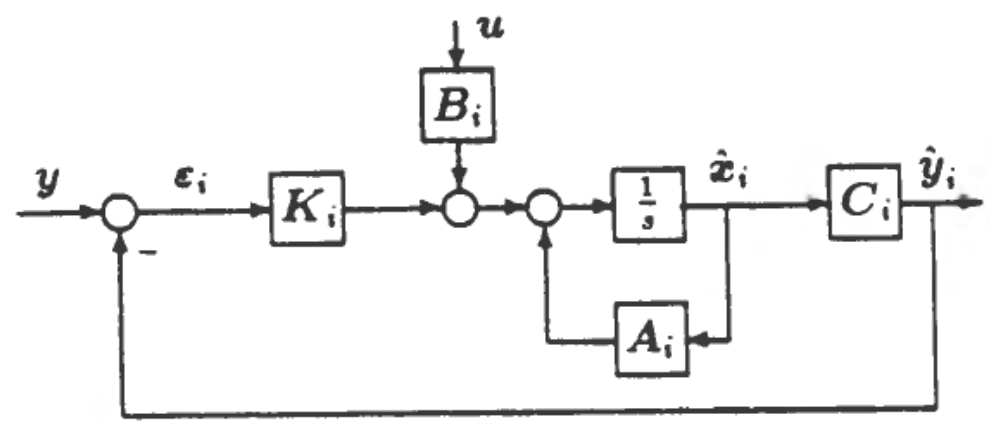

Figure 2. $\mathscr{F}\left(M_{i}, K_{i}\right)$ viewed as a proportional feedback system.

\section{A trade-off problem in MMAE}

This section highlights the model discrimination versus tracking properties of MMAE. By tracking is meant the ability of a filter to predict the output $y\left(t_{k}\right)$ given $Y_{k-1}$. The trade-off problem may be stated as follows: On one hand, we want good tracking capabilities for each filter $\mathscr{F}\left(M_{i}, K_{i}\right)$, when $S^{*}=S_{i \cdot}$ On the other, we want the residuals to be distant, to achieve fast and reliable model discrimination. The side in the trade-off which is favoured, depends a lot on how strongly each filter updates its state estimates from the measurements $y\left(t_{k}\right)$. To illustrate this, let the system operate in only two modes, $S^{*} \in\left\{S_{1}, S_{2}\right\}$, with the corresponding models $M_{1}$ and $M_{2}$. Also restrict the models to be linear with continuous time measurements.

$$
\begin{aligned}
\frac{d}{d t} x_{i}(t) & =A_{i} x_{i}(t)+B_{i} u(t)+v_{i}(t) \\
y_{i}(t) & =C_{i} x_{i}(t)+w(t)
\end{aligned}
$$

Here $\boldsymbol{A}_{i}=\boldsymbol{A}\left(\boldsymbol{\theta}_{i}\right)$ and $\boldsymbol{C}_{i}=\boldsymbol{C}\left(\boldsymbol{\theta}_{i}\right)$. Then $\mathscr{F}\left(M_{1}, \boldsymbol{K}_{1}\right)$ and $\mathscr{F}\left(M_{2}, \boldsymbol{K}_{2}\right)$ can be viewed as feedback systems with proportional gain as shown in Fig. 2. Introduce $y\left(t \mid S_{i}\right)$ as the measurement from $S^{*}$ when known to be in mode $S_{i}$ and similarly $y\left(\mathrm{j} \omega \mid S_{i}\right)$ in the frequency domain. Then from Fig. 2

$$
\begin{aligned}
\boldsymbol{\varepsilon}_{i}\left(\mathrm{j} \omega \mid \boldsymbol{S}_{j}\right)= & {\left[\boldsymbol{I}+\boldsymbol{P}_{i}(\mathrm{j} \omega) \boldsymbol{K}_{i}\right]^{-1} \boldsymbol{y}\left(\mathrm{j} \omega \mid S_{j}\right) } \\
& -\left[\boldsymbol{I}+\boldsymbol{P}_{i}(\mathrm{j} \omega) \boldsymbol{K}_{i}\right]^{-1} \boldsymbol{P}_{i}(\mathrm{j} \omega) \boldsymbol{B}_{i} \boldsymbol{u}(\mathrm{j} \omega) \\
\boldsymbol{P}_{i}(\mathrm{j} \omega)= & \boldsymbol{C}_{i}\left(\mathrm{j} \omega I-\boldsymbol{A}_{i}\right)^{-1}, \quad i, j=1,2
\end{aligned}
$$

Assume that both $M_{1}$ and $M_{2}$ are stochastic observable and stochastic controllable (Maybeck 1979, Chap. 5). Then for increasing process noise covariance matrices $V_{1}$ and $V_{2}$, both filters have the same property: $\hat{y}_{i}(\mathrm{j} \omega) \rightarrow y(j \omega)$ and $\varepsilon_{i}(\mathrm{j} \omega) \rightarrow 0$ regardless of the mode. This behaviour may give the situation of Eqn. (8) and the filters mask the differences between $M_{1}$ and $M_{2}$. This indicates that the distance, in some sense, between the residuals is crucial. One possible choice is to define

$$
\begin{aligned}
\varepsilon_{i j}(t) & =\varepsilon_{i}(t)-\varepsilon_{j}(t), \quad i \neq j \\
\varepsilon_{i}(t) & =y(t)-y_{i}(t)
\end{aligned}
$$

and use some vector norm $\left\|\varepsilon_{i j}\right\|$ as the distance between any two residuals. Denote $\left\|\varepsilon_{i j}\right\|$ as the Inter-Residual Distance and the vector $\varepsilon_{i j}\left(t_{k}\right)$ as the Inter-Residual Difference. When filter gains become large, we have that 


$$
\varepsilon_{i j}(t)=\hat{y}_{j}(t)-\hat{y}_{i}(t) \rightarrow 0
$$

which highlights the fact that the filters in the bank should not be tuned totally independently.

\section{Filter gain modulation}

A method for modulating the filter gains according to a measure of $\varepsilon_{i j}, i \neq j$ is now proposed. A simple quadratic form

$$
J_{i j}(t)=\varepsilon_{i j}^{\mathrm{T}}(t) \Gamma_{i j} \varepsilon_{i j}(t), \quad i \neq j
$$

is chosen as the distance measure of $\varepsilon_{i j}$, where $\Gamma_{i j}$ is a positive definite diagonal scaling matrix. The number of filters is here restricted to $N=2$ but extensions to more filters is outlined at the end of this chapter. The main principle of the method is to keep the interresidual distance measure $J_{12}(t)$ above a specified limit $J_{12}^{0}$ by adjusting the filter gains. A general way to achieve this is by varying the process noise covariances $V_{1}$ and $V_{2}$. In filter calculations $V_{i}$ is now replaced by modulated process noice covariance matrices $V_{i}(t)$ defined as

$$
V_{i}^{\prime}(t)=\eta(t) V_{i}, \quad i=1,2
$$

where $\eta(t) \in\left[\eta_{\min }, 1.0\right]$ and the lower bound $\eta_{\min }$ can be chosen to give a lower bound on $V_{i}^{\prime}(t)$. The restriction $\eta_{\min }>0$, makes $V_{i}^{\prime}(t) \geqslant 0$ and the upper bound $\eta \leqslant 1 \cdot 0$ is chosen to secure that $V_{i}^{\prime}(t) \leqslant V_{i}(t)$.

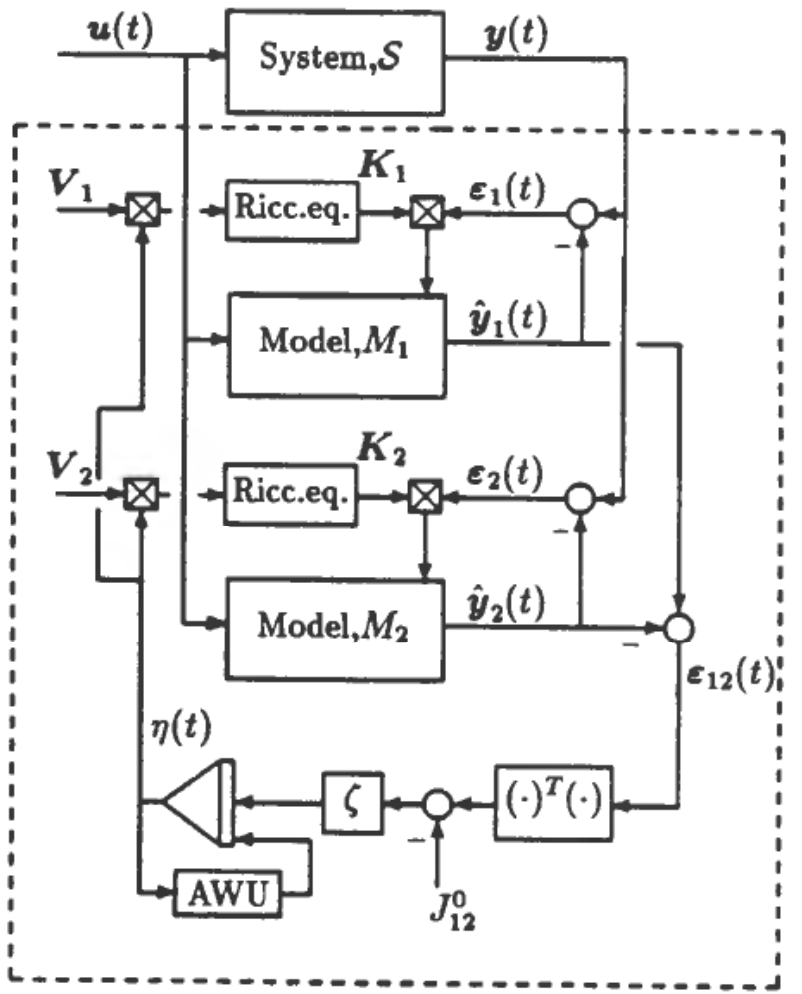

Figure 3. Modulation of process noise covarance. AWU is anti-integration windup, see Eqn. (14). 


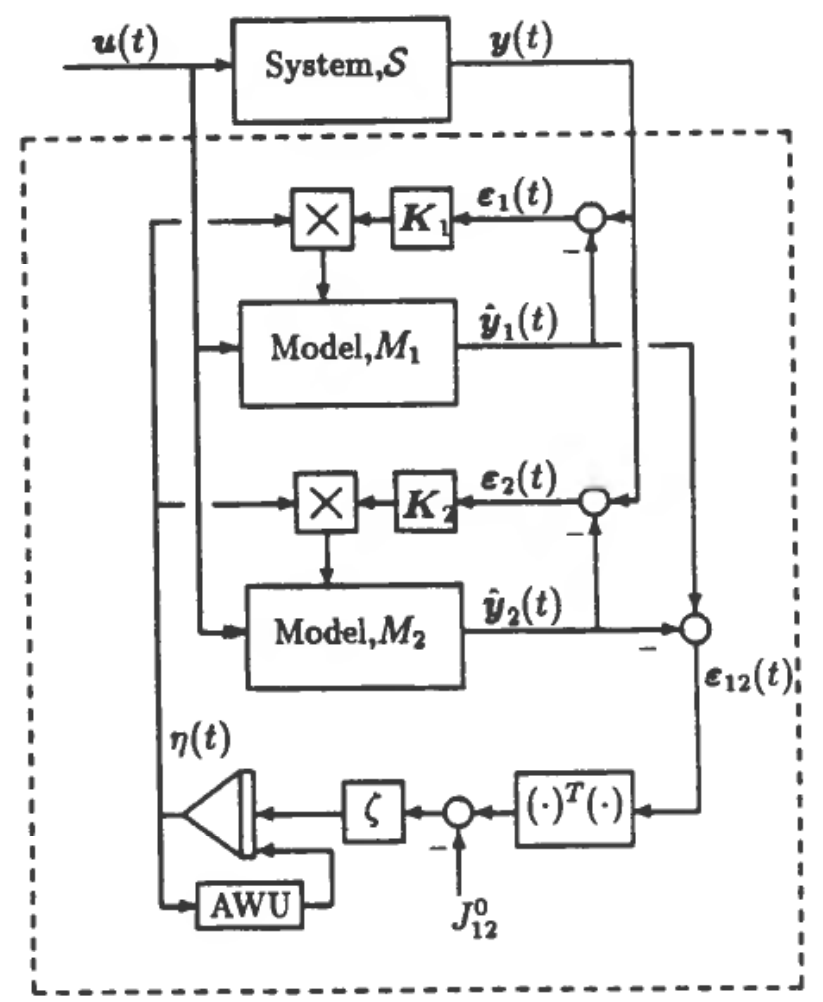

Figure 4. Modulation of new information in the filters. AWU is anti-integration windup, see Eqn. (14).

The time derivative of the modulating variable $\eta(t)$, should be an odd function of $J_{12}(t)-J_{12}^{0}$, being zero for $J_{12}(t)=J_{12}^{0}$ and for $\eta \notin\left\langle\eta_{\min }, 1 \cdot 0\right\rangle$. One choice is

$$
\frac{d}{d t} \eta(t)= \begin{cases}\zeta\left[J_{12}(t)-J_{12}^{0}\right], & \text { Cond } 1 \\ 0 & , \text { Cond } 2\end{cases}
$$

where the conditions in Eqn. (14) are:

$$
\begin{aligned}
& \text { Cond 1: } \eta \in\left\langle\eta_{\min }, 1 \cdot 0\right\rangle \\
& \text { Cond 2: }\left\{\begin{array}{l}
\left(\eta=\eta_{\min } \text { AND } \zeta\left[J_{12}(t)-J_{12}^{0}\right]<0\right) \text { OR } \\
\left.\eta=1 \cdot 0 \quad \text { AND } \zeta\left[J_{12}(t)-J_{12}^{0}\right]>0\right)
\end{array}\right.
\end{aligned}
$$

These conditions provides anit-integration windup (AWU), see Figs. 3 and 4. The constant $\zeta>0$, must be specified together with the lower inter-residual distance limit $J_{12}^{0}$ or a lower inter-residual difference limit $\varepsilon_{12}^{0}$, such that $J_{12}^{0}=\varepsilon_{12}^{0 \mathrm{~T}} \Gamma_{12} \varepsilon_{12}^{0}$. Note that Eqn. (14) is an integrator and $\zeta$ should be selected to provide proper attenuation of noise on $\eta(t)$. The concept is shown in Fig. 3 for $N=2$. From the standard Kalman filter equations it follows that increasing values of $V_{1}$ and $V_{2}$ increase the filter gains. This in turn, reduces the value of $J_{12}$. Small values of $V_{1}$ and $V_{2}$ make $J_{12}$ more dependent on the differences between $M_{1}$ and $M_{2}$, and presumably greater in mean square sense. Eqns. (12), (13) and (14) adjust $\eta(t)$ to an equilibrium in mean value such that $J_{12}(t)$ $=J_{12}^{0}$. This is shown inside the dashed lines in Fig. 3 and described by Eqns. (15) to (19). 
These equations constitute a system of nonlinear differential equations ((15) to (19)) of order $n^{2}+3 n+1$ knowing that the state estimate covariance matrix $\boldsymbol{X}_{i}(t)=\boldsymbol{X}_{i}^{\mathrm{T}}(t)$.

$$
\begin{aligned}
& \frac{d}{d t} \hat{x}_{1}(t)=\left[A_{1}-X_{1}(t) C_{1}^{\mathrm{T}} W^{-1} C_{1}\right] \hat{x}_{1}(t) \\
& +X_{1}(t) C_{1}^{\mathrm{T}} \boldsymbol{W}^{-1} y(t)+B_{1} u(t) \\
& \frac{d}{d t} \hat{\boldsymbol{x}}_{2}(t)=\left[A_{2}-X_{2}(t) C_{2}^{\mathrm{T}} \boldsymbol{W}^{-1} C_{2}\right] \hat{\boldsymbol{x}}_{2}(t) \\
& +X_{2}(t) C_{2}^{\mathrm{T}} \boldsymbol{W}^{-1} y(t)+B_{2} u(t) \\
& \frac{d}{d t} X_{1}(t)=A_{1} X_{1}(t)+X_{1}(t) A_{1}^{\mathrm{T}} \\
& -X_{1}(t) C_{1}^{\mathrm{T}} W^{-1} C_{1} X_{1}(t)+\eta(t) V_{1} \\
& \frac{d}{d t} \boldsymbol{X}_{2}(t)=A_{2} \boldsymbol{X}_{2}(t)+\boldsymbol{X}_{2}(t) A_{2}^{\mathrm{T}} \\
& -X_{2}(t) C_{2}^{\mathrm{T}} \boldsymbol{W}^{-1} C_{2} X_{2}(t)+\eta(t) V_{2} \\
& \frac{d}{d t} \eta(t)=\left\{\begin{array}{cc}
\zeta\left[\left(C_{2} \hat{\boldsymbol{x}}_{2}(t)-C_{1} \hat{\boldsymbol{x}}_{1}(t)\right)^{\mathrm{T}} \boldsymbol{\Gamma}_{12}\right. & \\
\left.\left(C_{2} \hat{\boldsymbol{x}}_{2}(t)-C_{1} \hat{\boldsymbol{x}}_{1}(t)\right)-J_{12}^{0}\right], & \text { Cond 1 } \\
0, & \text { Cond 2 }
\end{array}\right.
\end{aligned}
$$

Since $V_{i}^{\prime}(t)$ is a function of time, filter gains cannot be precomputed even for linear models.

A simplification of the method described above is obtained if, instead of modulating $V_{i}$, the new information $\boldsymbol{K}_{i} \varepsilon_{i}(t)$, is modulated as

$$
K_{i}^{\prime}(t) \varepsilon_{i}(t)=\eta(t) K_{i} \varepsilon_{i}(t), \quad i=1,2, \quad \eta(t) \in\left[\eta_{\min }, 1 \cdot 0\right]
$$

The individual filter gains $\boldsymbol{K}_{\boldsymbol{i}}$ are not precomputable, and only the modulation is computed on-line. This simplified method is shown inside the dashed lines of Fig. 4 and described by Eqns. (19), (21) and (22).

$$
\begin{aligned}
\frac{d}{d t} \hat{x}_{1}(t)= & \left(A_{1}-\eta(t) K_{1} C_{1}\right) \hat{x}_{1}(t) \\
& +\eta(t) K_{1} y(t)+B_{1} u(t) \\
\frac{d}{d t} \hat{x}_{2}(t)= & \left(A_{2}-\eta(t) K_{2} C_{2}\right) \hat{x}_{2}(t) \\
& +\eta(t) K_{2} y(t)+B_{2} u(t)
\end{aligned}
$$

Eqns. (21) and (22) together with (19) constitute a $2 n+1$ dimensional nonlinear statespace system with $u(t), y(t)$ and $J_{12}^{0}$ as inputs. It should be noticed that in general, modulation of $K_{i} \varepsilon_{i}$ as in Eqn. (20) and modulation of $V_{i}$ as in Eqn. (13) does not give the same filter gain. This is due to the filter gain calculation in Eqn. (23) together with the Riccati equations (17) and (18).

$$
K_{i}(t)=X_{i}(t) C_{i}^{\mathrm{T}} W^{-1}
$$


The question of stability and which constraints this imposes on $\eta(t)$ and $\zeta$ is now considered. As can be seen from Eqns. (15) to (18) and Eqns. (21) and (22), analysis is simpler when modulating $K_{i} \varepsilon_{i}$ directly, because the Riccati equations are eliminated. For examination of stability we assume that the models are linear and by utilizing a Lyapunov function, we can establish a sufficient condition for asymptotic stability of the autonomous parts of the Eqns. (19), (21) and (22). We consider the Lyapunov function

$$
V(\hat{x})=\hat{\boldsymbol{x}}^{\mathrm{T}} \boldsymbol{Q} \hat{\boldsymbol{x}} \in \mathbb{R}
$$

where $Q=Q^{\mathrm{T}}>0$ and

$$
\tilde{\boldsymbol{x}}=\left[\begin{array}{c}
\hat{\boldsymbol{x}}_{1} \\
\boldsymbol{x}_{2} \\
\eta
\end{array}\right]
$$

Global asymptotic stability is guaranteed if

$$
\frac{d}{d t} V(\tilde{\boldsymbol{x}})=\left[\frac{d}{d t}\left(\tilde{\boldsymbol{x}}^{\mathrm{T}}\right) Q \tilde{\boldsymbol{x}}+\tilde{\boldsymbol{x}}^{\mathrm{T}} Q \frac{d}{d t}(\tilde{\boldsymbol{x}})\right]<0, \quad \forall \tilde{\boldsymbol{x}}
$$

We now make the rather restrictive and simplifying choice $\boldsymbol{Q}=\boldsymbol{I}$ and substitute Eqns. (19), (21) and (22) into Eqn. (26) which gives

$$
\begin{aligned}
\frac{d}{d t} V(\tilde{x})= & \hat{x}_{1}^{\mathrm{T}}\left\{A_{1}^{\mathrm{T}}+A_{1}-\eta\left(\left[K_{1} C_{1}\right]^{\mathrm{T}}+K_{1} C_{1}\right)\right\} \hat{x}_{1} \\
& +\hat{x}_{2}^{\mathrm{T}}\left\{A_{2}^{\mathrm{T}}+A_{2}-\eta\left(\left[K_{2} C_{2}\right]^{\mathrm{T}}+K_{2} C_{2}\right)\right\} \hat{x}_{2} \\
& +2 \zeta \eta\left\{\hat{x}_{1}^{\mathrm{T}} C_{1}^{\mathrm{T}} \Gamma_{12} C_{1} \hat{x}_{1}+x_{2}^{\mathrm{T}} C_{2}^{\mathrm{T}} \Gamma_{12} C_{2} \hat{x}_{2}\right. \\
& \left.-\hat{x}_{1}^{\mathrm{T}} C_{1}^{\mathrm{T}} \Gamma_{12} C_{2} \hat{x}_{2}-\hat{x}_{2}^{\mathrm{T}} C_{2}^{\mathrm{T}} \Gamma_{12} C_{1} \hat{x}_{1}\right\}
\end{aligned}
$$

Reorganize Eqn. (27) into

$$
\frac{d}{d t} V(\tilde{x})=-\left[\hat{x}_{1}^{\mathrm{T}}, \hat{\boldsymbol{x}}_{2}^{\mathrm{T}}\right] \boldsymbol{P}(\eta, \zeta)\left[\begin{array}{l}
\hat{\boldsymbol{x}}_{1} \\
\hat{\boldsymbol{x}}_{2}
\end{array}\right]
$$

where the symmetric matrix $\boldsymbol{P}(\eta, \zeta)$ is partitioned into the four submatrices

$$
\begin{aligned}
P_{11}(\eta, \zeta)= & -A_{1}^{\mathrm{T}}-A_{1}+\eta\left(\left[K_{1} C_{1}\right]^{\mathrm{T}}+K_{1} C_{1}\right) \\
& -2 C_{1}^{\mathrm{T}} \Gamma_{12} C_{1} \zeta \eta \\
P_{12}(\eta, \zeta)= & P_{21}^{\mathrm{T}}(\eta, \zeta)=2 C_{1}^{\mathrm{T}} \Gamma_{12} C_{2} \zeta \eta \\
P_{22}(\eta, \zeta)= & -A_{2}^{\mathrm{T}}-A_{2}+\eta\left(\left[K_{2} C_{2}\right]^{\mathrm{T}}+K_{2} C_{2}\right) \\
& -2 C_{2}^{\mathrm{T}} \Gamma_{12} C_{2} \zeta \eta
\end{aligned}
$$

Now, if a region $\mathscr{h}$ in the $\eta, \zeta$ plane can be found such that

$$
\mathscr{R}=\left\{\eta, \zeta \mid \boldsymbol{P}(\eta, \zeta)>\mathbf{0}, \eta \in\left[\eta_{\min }, 1 \cdot 0\right], \zeta>0\right\}
$$

then Eqn. (26) is satisfied and the system formed by the Eqns. (19), (21) and (22) is asymptotic stable for all $\eta, \zeta \in \mathscr{R}$. The region $\mathscr{R}$ may however be a conservative restriction on $\eta$ and $\zeta$. An approximation of $\mathscr{R}$ can be found numerically by partitioning the $\eta, \zeta$ plane into a grid and testing $\boldsymbol{P}(\eta, \zeta)$ for positive definiteness at all grid points. This is 
demonstrated for a simple system in the simulated example. Note that it is only necessary to analyse Cond 1 in Eqn. (19) by the Lyapunov function, since under Cond 2 stability is only concerned with the linear Eqns. (21) and (22). Stability analysis is more complicated when modulating $\boldsymbol{V}_{i}$ instead of modulating $\boldsymbol{K}_{i} \boldsymbol{\varepsilon}_{i}$, although there are some results which may be used. For stable linear models, both filters $\mathscr{F}\left(M_{1}, K_{1}\right)$ and $\mathscr{F}\left(M_{2}, K_{2}\right)$ are separately stable for all $\eta \in\left[\eta_{\min }, 1 \cdot 0\right]$ and for all $\zeta>0$ because $\eta V \geqslant 0$. See (Jazwinski 1970, pp. 234-244). This does however not guarantee asymptotic stability, because $\eta$ may oscillate within the interval $\left[\eta_{\min }, 1.0\right]$.

For Extended Kalman filters and higher orders filters there is no computational benefit in modulating the new information vectors rather than the process noise covariance matrices, because filter gains are computed on-line. Thus direct modulation of new information should only be considered for steady state filters. But even for steady state filters direct modulation of new information may introduce errors. Nonlinear models make Eqns. (15)-(19) even more intractable for analysis, and extensive simulations should be carried out in order to evaluate a bank of nonlinear filters with IRDF.

In order to use the concept of filter gain modulation for three or more models, one possible approach is to scan $\left(N^{2}-N\right) / 2$ distance measures and select $J_{\min }(t)=\min _{i, j} J_{i j}(t), i \neq j$. Then substitute $J_{12}(t)$ with $J_{\min }(t)$ and $J_{12}^{0}$ with an overall lower distance measure $J^{0}$ in Eqn. (14). Another method is to consider only the interresidual distance between the two most probable models. However this method suffers from the fact that one of the probabilities approach unity while the others become zero. This problem can be solved by reducing the time horizon of the probability calculation, (Magalhães and Binder, 1987).

\section{Simulation tests}

We shall demonstrate the IRDF concept by applying it on a second order linear SISO system $S^{*}$ given in a continuous discrete time formulation

$$
\begin{aligned}
\frac{d}{d t}\left[\begin{array}{l}
x_{1}(t) \\
x_{2}(t)
\end{array}\right] & =\left[\begin{array}{cc}
-0 \cdot 5 & 1 \\
0 & a
\end{array}\right]\left[\begin{array}{l}
x_{1}(t) \\
x_{2}(t)
\end{array}\right]+\left[\begin{array}{l}
0 \\
0 \cdot 9
\end{array}\right] u\left(t_{k}\right) \\
y\left(t_{k}\right) & =\left[\begin{array}{ll}
1 & 0
\end{array}\right]\left[\begin{array}{l}
x_{1}\left(t_{k}\right) \\
x_{2}\left(t_{k}\right)
\end{array}\right]
\end{aligned}
$$

Here $y\left(t_{k}\right)$ is discrete time measurement and $u\left(t_{k}\right)$ is control input changed only at discrete time instants $t_{k}$. The operational modes are determined by the parameter $a$, which has the values $a=0.5$ in mode $S_{1}$ and $a=1.0$ in mode $S_{2}$. The corresponding models $M_{1}$ and $M_{2}$ are given by

$$
\begin{gathered}
\frac{d}{d t}\left[\begin{array}{l}
\hat{x}_{1}(t) \\
\hat{x}_{2}(t)
\end{array}\right]_{i}=\left[\begin{array}{cc}
-0-5 & 1 \\
0 & -a
\end{array}\right]_{i}\left[\begin{array}{l}
\hat{x}_{1}(t) \\
\hat{x}_{2}(t)
\end{array}\right]_{i}+\left[\begin{array}{l}
0 \\
1
\end{array}\right] u\left(t_{k}\right) \\
+\left[\begin{array}{l}
v_{1}(t) \\
v_{2}(t)
\end{array}\right]_{i} \\
\hat{y}_{i}\left(t_{k}\right)=\left[\begin{array}{ll}
1 & 0
\end{array}\right]\left[\begin{array}{l}
\hat{x}_{1}\left(t_{k}^{-}\right) \\
\hat{x}_{2}\left(t_{k}^{-}\right)
\end{array}\right]_{i}+w\left(t_{k}\right)
\end{gathered}
$$

where $t \in\left[t_{k}, t_{k+1}\right\rangle, i=1,2$ and $\hat{x}_{i}\left(t_{k}^{-}\right)$is the estimate of $x_{i}(t)$ at sample instant $t_{k}$ before adding new information. The sample interval is 0.1 time units. Note that the control 


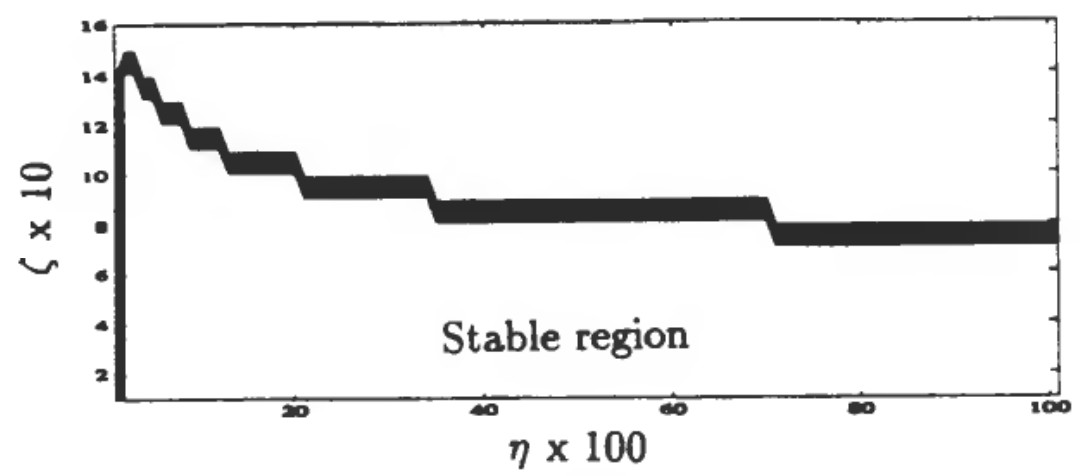

Figure 5. Approximated stability region in the $\eta, \zeta$ plane. See Eqn. (29).

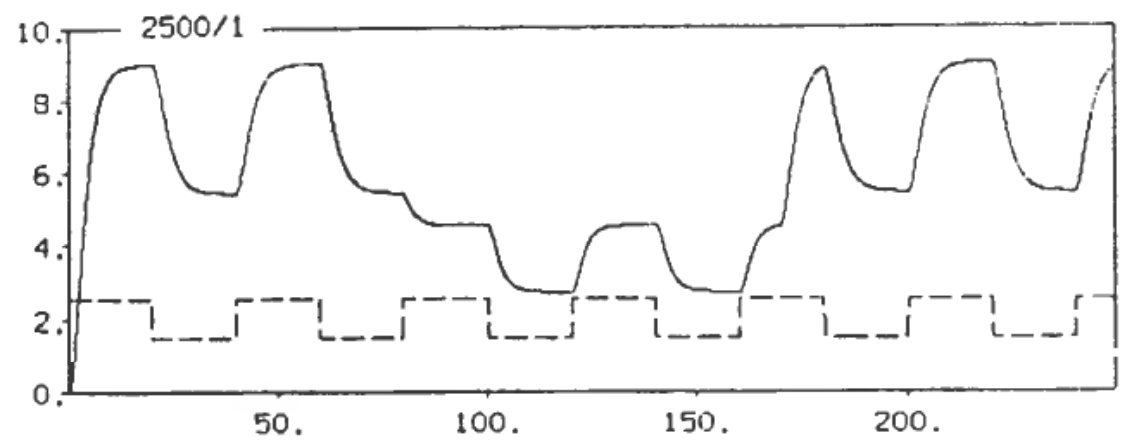

Figure 6. System output $y\left(t_{k}\right)$ (solid line) and input $u\left(t_{k}\right)$ (dashed line).

input matrix in Eqn. (30) differs $10 \%$ from the one in Eqn. (31). Hence, $S^{*}$ is not a member in $\mathscr{M}$. In this example we shall apply direct modulation of filter gains, $\boldsymbol{K}_{i}=\left[k_{1} k_{2}\right]_{i}^{\mathrm{T}}$, as shown in Fig. 4. An approximation of the stability region $\mathscr{R}$ given by Eqn. (29) is shown in Fig. 5. Here the scaling matrix is a scalar and chosen as $\Gamma_{12}=1$. The stable region corresponds to $\boldsymbol{P}(\eta, \zeta)>0$ and the other region to $\boldsymbol{P}(\eta, \zeta) \leqslant 0$. By choosing $\eta_{\min }=0$, asymptotic stability is guaranteed for $\eta \in[0,1 \cdot 0]$ and $\zeta \in\langle 0,0 \cdot 7]$. In simulations $\zeta$ is chosen to 0.5 if no other value is stated. For small values of $\eta ; \zeta$ may be larger than 0.5 , still not violating the stability constraint. This stable region is obtained when $K_{1}=[0.92,0.55]^{\mathrm{T}}, \boldsymbol{K}_{2}=[0.92,0.36]^{\mathrm{T}}$ and when the process and measurement noise have covariances $V_{1}=V_{2}=10 I$ and $W=0.1$ respectively. In all simulations, the system and the filters were excited by a square wave $u\left(t_{k}\right)$ with amplitude $=0.5$, mean $=2.0$ and period $=40.0$, all being dimensionless quantities. Excitation $u\left(t_{k}\right)$ and system output $y\left(t_{k}\right)$ are shown in Fig. 6.

The Kalman filters were implemented in continuous-discrete time formulation with measurement updating at every 0-1 time unit. Between two successive sample instants $t_{k}$ and $t_{k+1}$, both the system and filter state equations are integrated numerically using an explicit variable step length Runge-Kutta method of order 5(4). The operational mode changes from $S_{1}$ to $S_{2}$ at 80 time units and back to $S_{1}$ at 170 time units. Initial values of the system were $x(0)=\hat{\boldsymbol{x}}_{1}(0)=\hat{\boldsymbol{x}}_{2}(0)=0$ and $\eta(0)=1 \cdot 0$. The residuals obtained without IRDF are compared to those obtained with IRDF $(\zeta=0-5)$ in Figs. 7 and 8 . For clarity, no measurement noise was added to the system output here. The lower inter-residual difference limit was specified to $\varepsilon_{12}^{0}=0 \cdot 3$. Figure 9 shows $\varepsilon_{12}$ with and without IRDF. 


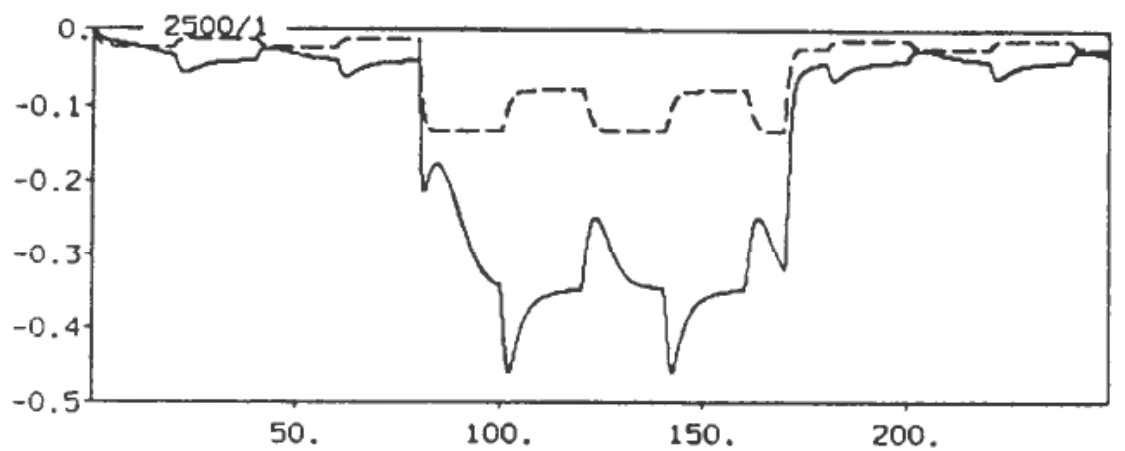

Figure 7. Residual $\varepsilon_{1}$ of $\mathscr{F}\left(M_{1}, K_{1}\right)$ with (solid line) and without (dashed line) IRDF.

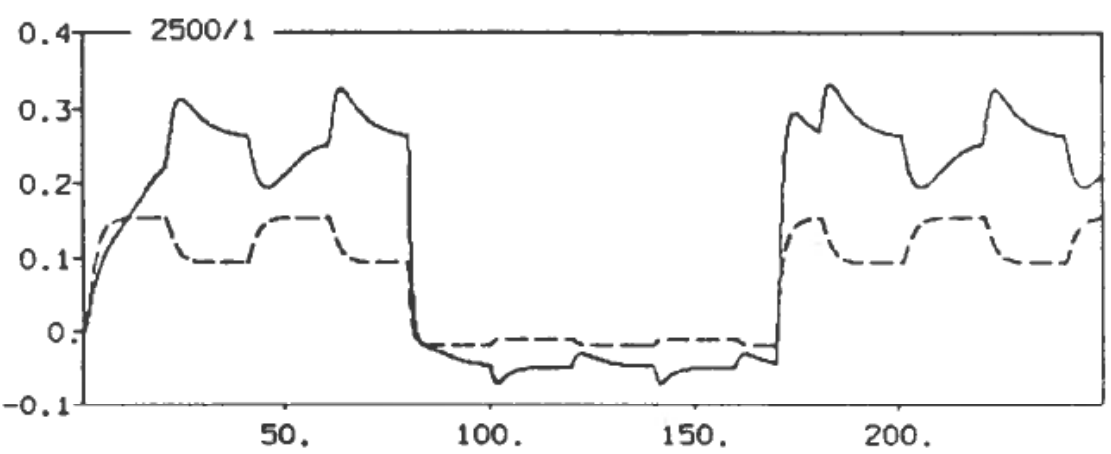

Figure 8. Residual $\varepsilon_{2}$ of $\mathscr{F}\left(M_{2}, K_{2}\right)$ with (solid line) and without (dashed line) IRDF.

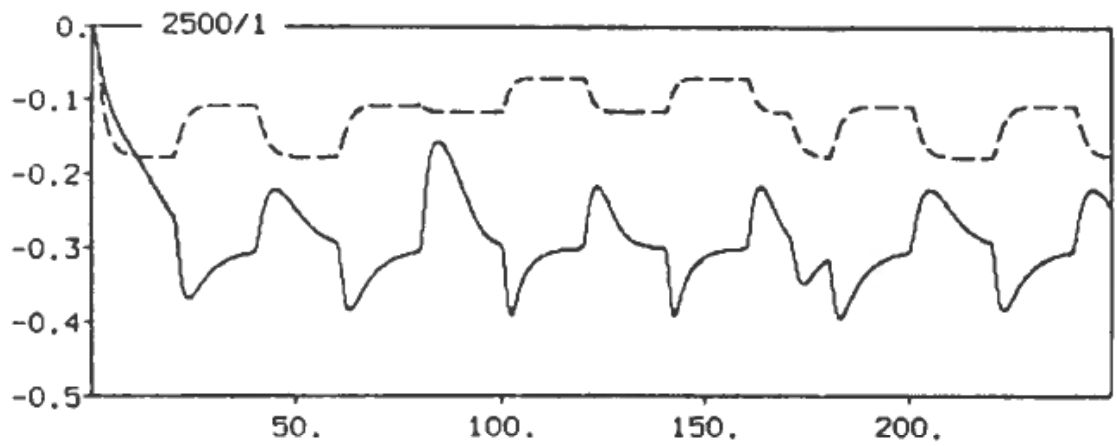

Figure 9. Inter-residual difference $\varepsilon_{12}$ with (solid line) and without (dashed line) IRDF.

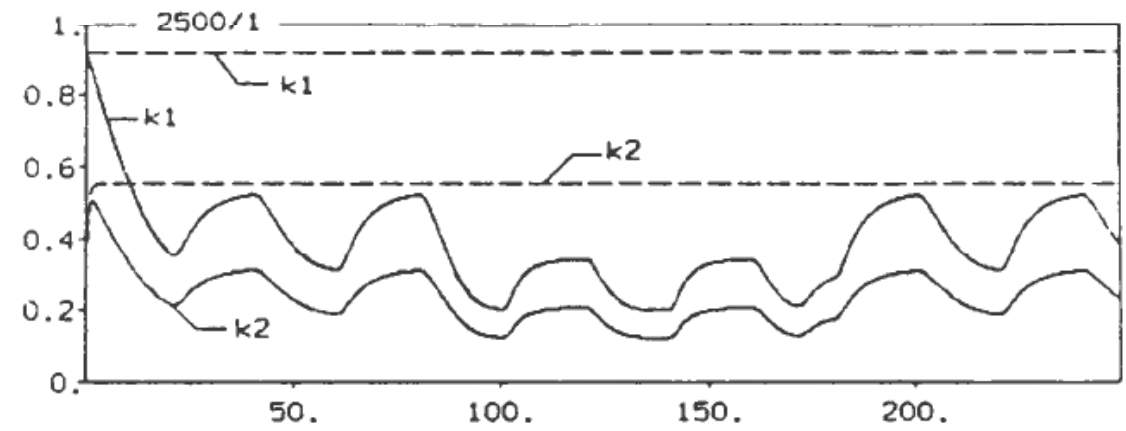

Figure 10. Filter gains $k_{1}$ and $k_{2}$ of $\mathscr{F}\left(M_{1}, K_{1}\right)$ with (solid line) and without (dashed line) IRDF. 


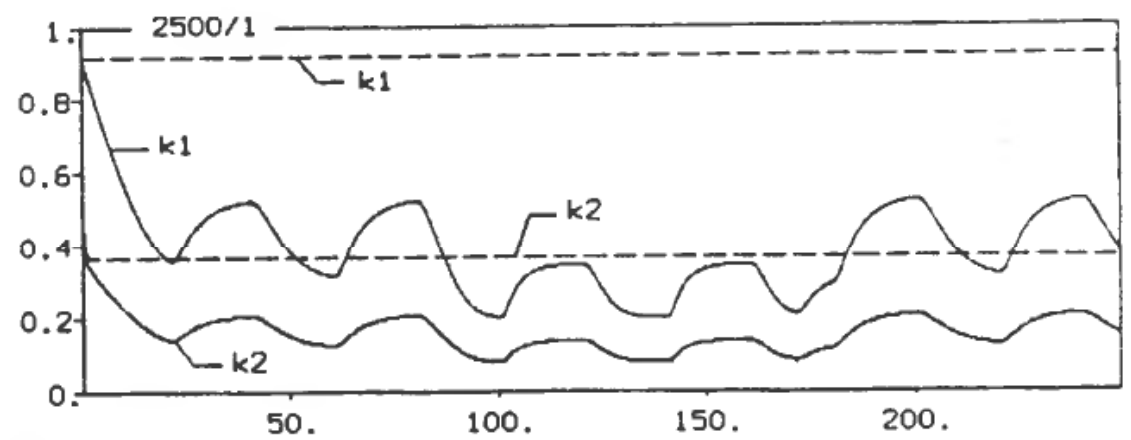

Figure 11. Filter gains $k_{1}$ and $k_{2}$ of $\mathscr{F}\left(M_{2}, K_{2}\right)$ with (solid line) and without (dashed line) IRDF.

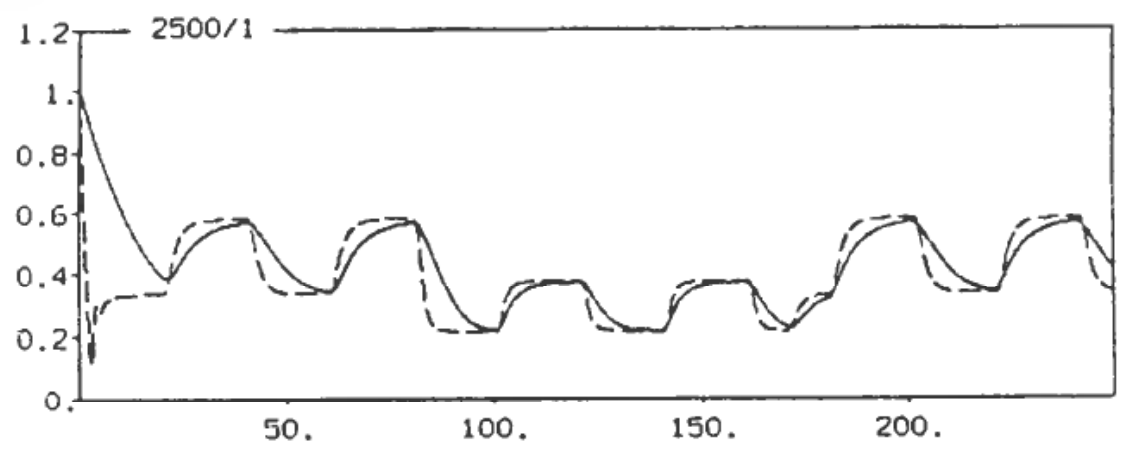

Figure 12. Modulating variable $\eta(t)$, for $\zeta=0.5$ (solid line) and for $\zeta=4.0$ (dashed line).

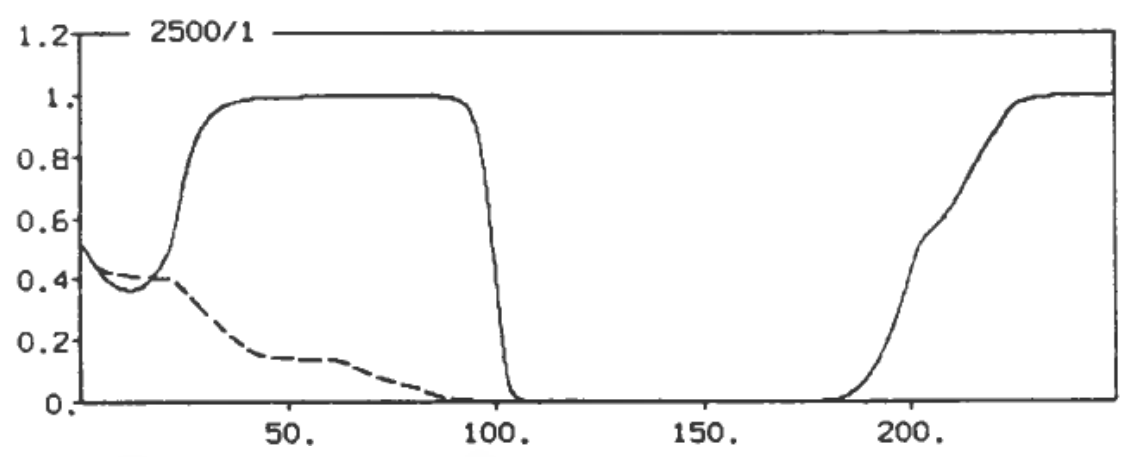

Figure 13. Probability $P_{1}$ of $M_{1}$, with (solid line) and without (dashed line) IRDF. $\left(P_{2}=1-P_{1}\right)$.

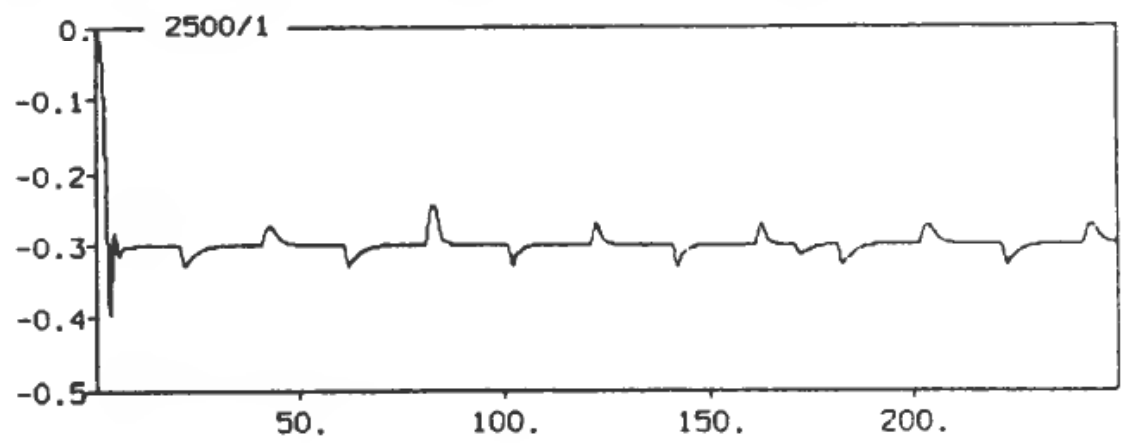

Figure 14. Residual difference $\varepsilon_{12}$, with IRDF and $\zeta=4 \cdot 0$. 
Observe that $\varepsilon_{12}$ varies around $-0 \cdot 3$ instead of 0-3 which is irrelevant due to Eqn. (12). The variation in $\varepsilon_{12}$ is induced by the excitation, $u\left(t_{k}\right)$. Filter gains $k_{1}$ and $k_{2}$ are shown for both cases in Figs. 10 and 11, and $\eta$ is shown in Fig. 12. The figs. 7, 8 and 9 illustrate the trade-off problem.

A lower limit of $P_{i}\left(t_{k}\right)=0.001$ was applied in order to avoid that probability of the invalid model lock on to zero. The probability of $M_{1}$ is shown in Fig. 13 with and without IRDF. Initial probabilities were $P_{1}(0)=0-5$. Without the IRDF $P_{1}\left(t_{k}\right)$ is seen to approach zero despite $M_{1}$ is expected to be closest to $S^{*}$ before 70 time units. Also the change of mode at 170 time units is not detected without IRDF. This is due to the small difference between $\varepsilon_{1}\left(t_{k}\right)$ and $\varepsilon_{2}\left(t_{k}\right)$, which was denoted as irregular residual behaviour. However, with the IRDF, the residuals behave in a regular way and the probabilities become right.

In order to examine the influence of $\zeta$, simulations were carried out for $\zeta$ outside the stability region, $\mathscr{R}$. For $\zeta=4 \cdot 0$, Fig. 12 shows $\eta\left(t_{k}\right)$ and Fig. 14 shows $\varepsilon_{12}\left(t_{k}\right)$. The other parameters are unchanged. The variation in $\varepsilon_{12}$ about $\varepsilon_{12}^{0}$ is now smaller and $\eta$ adjusts faster than for $\zeta=0-5$. However the stability is maintained, which indicates that the stability region of Fig. 5 is rather conservative.

Simulations with zero mean Gaussian noise of covariance 0.1 added to the system output were also carried out. For values of $\zeta$ inside the stability region in Fig. 5, IRDF behaved similarly as without noise. Modulation of $\boldsymbol{V}_{\boldsymbol{i}}$ has also been simulated and similar results were observed for this system.

\section{Conclusions}

A method for on-line modulation of the filters used for Multiple Model Adaptive Estimation has been proposed. Stability of the method was investigated for a bank of two linear filters, and simulations were carried out using a second order SISO system to demonstrate the properties of the proposed method. The Inter-Residual Distance Feedback was a successful method to enhance the model discrimination properties of MMAE. Further investigations should include other distance measures between the models especially in a probabilistic sense. Also some means of determining $\eta_{\min }$ from an overall tracking capability of the filterbank should be considered. The concept of MMAE with IRDF extends naturally to discrimination between nonlinear models with different structures and different orders, only the dimensions of measurement vectors should be equal.

\section{ACKNOWLEDGMENTS}

This research has been supported by a scholarship from the Norwegian Institute of Technology received by the first author and by the Royal Norwegian Council for Scientific and Industrial Research (NTNF) through the MIP program.

\section{REFERENCES}

ANDERSON, B. D. O. and MoORE, J. B. (1979). Optimal Filtering (Prentice-Hall Inc., Englewood Cliffs, N.J.) Chap. 10, pp. 267-287.

Athans, M. and Chang, C. B. (1976) Adaptive Estimation and Parameter Identification Using Multiple Model Estimation Algorithm, Tech. Note 1976-28, Lincoln Lab., Lexington, Massachusetts. 
Athans, M., Castañon, D., Dunn, K., Greene, C. S., Lee, W. H., Sandell, N. R., and Willsky, A. S. (1977). The stochastic control of the F-8C aircraft using a multiple model adaptive control (MMAC) method-part I: equilibrium flight. IEEE Trans. Autom. Control, 22, 768-780.

GelB, A. (1984). Applied Optimal Estimation, 8th ed. (MIT Press, Cambridge, Massachusetts). JAZWINSKI, A. H. (1970). Stochastic Processes and Filtering Theory (Academic Press, New York). LAINIOTIS, D. G. (1971). Optimal adaptive estimation: structure and parameter adaption. IEEE Trans. Autom. Control, 16, 160-170.

Magalhães, M. F. and Binder, Z. (1987). A true multimodel estimation algorithm. Prepr. 10th IFAC World Congr. on Autom. Control, Munich, Germany, July 27-31, Vol. 10, pp. 260-264.

MAGILL, D. T. (1965). Optimal adaptive estimation of sampled stochastic processes. IEEE Trans. Autom. Control, 10, 434-439.

MAYBECK, P. S. (1979). Stochastic Models, Estimation, and Control, Vol. 1 (Academic Press, New York).

MAYBECK, P. S. (1982). Stochastic Models, Estimation and Control, Vol. 2 (Academic Press, New York).

MAYBECK, P. S. and Pogoda, D. L. (1989). Multiple Model Adaptive Controller for the STOL F-15 with Sensor/-Actuator Failures, Proc. of the 28th IEEE Conf. on Dec. and Control, Tampa, Florida, pp. 1566-1572. 